\title{
Immunophysiology of experimental food allergy
}

\author{
MC Berin ${ }^{1,2}$ and L Mayer ${ }^{2}$
}

\begin{abstract}
Animal models of food allergy have been used to identify mechanisms involved in the development of sensitization to food proteins as well as immunologic mechanisms of adverse reactions to allergen reexposure. To counteract the normal tolerant responses to antigen generated in the gastrointestinal tract, investigators have used mucosal adjuvants or manipulated the mucosal barrier, taken advantage of endogenous adjuvanticity of some food allergens, or bypassed the oral route and sensitized through the skin. Site of antigen uptake in the gastrointestinal tract is a critical factor in both sensitization and anaphylaxis, and antigen uptake can be facilitated by immunoglobulin-E (IgE)-antigen complexes binding to CD23 on the epithelial cell surface. Studies on systemic anaphylaxis or local gastrointestinal manifestations of food allergy in mice have highlighted the contribution of IgE, mast cells, and pathogenic Th2 lymphocytes in experimental food allergy.
\end{abstract}

\section{INTRODUCTION}

Animal models of food allergy have been used to study both the inductive phase (mechanisms of sensitization) and the effector phase (pathophysiological mechanisms) of food allergic disease. Early studies were primarily performed using mice or rats sensitized to antigen systemically with adjuvant (alum, pertussis vaccine or pertussis toxin, or complete Freund's adjuvant). These models result in production of antigen-specific immunoglobulin (IgE) and hypersensitivity responses upon allergen challenge, and therefore they are useful for the study of effector mechanisms of food allergy. Due to their artificial sensitization method they have limited use for the study of the inductive phase of food allergy. A longstanding goal of food allergy researchers has been to develop adjuvant-free animal models of allergic sensitization that are responsive to oral rechallenge with allergen, resulting in immediate hypersensitivity symptoms representative of human disease. One major challenge to this goal is that the normal immune response to antigens encountered via the gastrointestinal tract is a response of active immune tolerance. Oral exposure results in the generation of food antigen-specific $\mathrm{T}$ lymphocytes with regulatory activity. These regulatory cells suppress the generation of antigen-specific effector lymphocyte responses, including pro-allergic Th2 responses and IgE production. ${ }^{1}$ Studies with human subjects have shown that oral tolerance is impaired in patients with inflammatory bowel disease, ${ }^{2}$ which may contribute to inappropriate immune reactivity to the commensal flora. Although this has not been conclusively shown in food allergy, presumably a lack of oral tolerance is also an underlying factor in food allergic disease. More recently described animal models have taken several approaches to break normal oral tolerance pathways: the use of mucosal adjuvants, and manipulation of epithelial barrier function by different means. In addition, some food allergens have innate adjuvant activity that may promote sensitization in the absence of an experimental break in tolerance. And finally, the normal tolerance response in the gut has led some investigators to the idea that sensitization through other routes (i.e., the skin) may be more relevant to sensitization in human food-allergic disease.

\section{BREAKING TOLERANCE:THE INDUCTIVE PHASE OF FOOD ALLERGY \\ Adjuvants}

Cholera toxin (CT) is a potent mucosal adjuvant that abrogates oral tolerance to coadministered antigens. ${ }^{3}$ Snider et al. ${ }^{4}$ showed that oral administration of CT with the antigens hen egg lysozyme or ovalbumin (OVA) resulted in antigen-specific IgE production, and anaphylaxis when the mice were rechallenged systemically with antigen. In addition, intestinal hypersensitivity responses were detected in mice sensitized using CT as adjuvant, demonstrating the potential for responsiveness to oral reexposure. Li et al. ${ }^{5,6}$ subsequently modified this protocol to generate models of milk- and peanut-induced anaphylaxis. Multiple feeds of antigen plus CT resulted in allergen-specific IgE production, and when mice were orally rechallenged, they underwent systemic anaphylaxis characterized by histamine release and drop in body temperature. This approach has now been used to

\footnotetext{
1Jaffe Food Allergy Institute, Mount Sinai School of Medicine, New York, New York, USA; ${ }^{2}$ Immunology Institute, Mount Sinai School of Medicine, New York, New York, USA. Correspondence: MC Berin (cecilia.berin@mssm.edu)

Received 16 September 2008; accepted 30 September 2008; published online 29 October 2008. doi:10.1038/mi.2008.72
} 
sensitize mice to buckwheat, ${ }^{7}$ lupin proteins, ${ }^{8}$ milk or individual milk proteins, ${ }^{6,9-11}$ and peanut or peanut allergens. ${ }^{5,12-15}$

Understanding the mechanisms by which CT can break mucosal tolerance is of interest as the same pathways may be playing a role in human food allergy. Administration of antigen plus the B subunit of CT does not lead to allergic sensitization, ${ }^{4}$ indicating that the enzymatic activity of the A subunit is likely important. However the target cell is not completely understood. Snider et al. ${ }^{4}$ showed that administration of CT together with antigen did not lead to enhanced uptake of antigen into the systemic circulation. Most adjuvants are believed to act primarily via innate pathways to cause maturation of antigen presenting cells. In vitro studies support this concept, in that macrophages and dendritic cells (DCs) mature and upregulate MHC class II and costimulatory molecules in response to CT. ${ }^{16-18}$ Administration of CT to mice induces an upregulation of interleukin (IL)-1 locally within the intestinal tissue, and exogenous administration of IL-1 can induce productive immune responses to coadministered antigens in place of CT. ${ }^{18,19}$ In addition, neutralization of IL-1 can abrogate effects of CT on enhanced T-cell priming by antigen-presenting cells. ${ }^{20}$ It is not known if local IL-1 may be primarily responsible for CT-induced allergic sensitization, as most studies have focused on the induction of protective immune responses such as IgA secretion. However, the central role of IL-1 in alum-induced Th2 responses via activation of innate pathways in $\mathrm{DCs}^{21,22}$ suggests that IL-1 may indeed be an important factor in the induction of allergic sensitization to food proteins in the gastrointestinal tract.

Lamina propria DCs constitutively capture antigen in the tissues and traffic through the lymph to draining lymph nodes. ${ }^{23,24}$ It is not known if DCs capture soluble protein antigen directly from the lumen as has been shown for bacteria ${ }^{25,26}$ or if they acquire antigen that has already breached the epithelial barrier. Oral administration of CT leads to the enhanced migration and maturation of gastrointestinal DCs from the lamina propria, to the MLN, where they present captured and processed antigen to T lymphocytes. ${ }^{27,28}$ In the Peyer's patch, CT also induces migration of DCs from the subepithelial dome to the T-cell area. ${ }^{29,30}$ Thus one major effect of CT is to facilitate presentation of antigen to $\mathrm{T}$ cells. We recently observed that in addition to conventional maturation, CT induces the upregulation of the costimulatory molecules OX40L and Jagged2 in DCs migrating from the lamina propria to the MLN. ${ }^{27}$ These molecules are known to facilitate differentiation of responder $\mathrm{T}$ cells into Th2 cells ${ }^{31-36}$ and we observed that neutralization of OX40L alone could abolish the enhanced Th2-priming activity of DCs draining from the small intestine. ${ }^{27}$ Despite the in vitro effects of Jagged2 on Th2 skewing, abolishment of Jagged2 has no effect on Th2 polarization in vivo, ${ }^{37}$ suggesting a dominant role for OX40L. Recently it was demonstrated that transfer of bone marrow DCs cultured with peanut antigen plus $\mathrm{CT}$ to naive mice could sensitize the recipient mice such that oral reexposure to peanut triggered local mast-cell degranulation. ${ }^{38}$ Peanut and CT treatment upregulated the expression of the molecule TIM-4 on DCs, and RNAi against TIM-4 in DCs or blocking antibodies against TIM- 1 on T cells abrogated sensitization in recipient mice. ${ }^{38,39}$ Factors promoting the upregulation of these Th2-inducing, tolerance-inhibiting costimulatory molecules on gastrointestinal DCs may therefore play an important role in allergic sensitization to foods.

CT is a widely used tool for experimental food allergy, but this bacterial toxin is unlikely to play a role in sensitization in human food allergic disease. However other more common microbial products also have adjuvant activity when coadministered with antigens. Yang et al. ${ }^{39}$ used the enterotoxin B from Staphylococcus aureus (SEB) to sensitize mice to OVA. Coadministration of SEB plus OVA resulted in upregulation of TIM- 4 on gastrointestinal DCs, and blockade of TIM- 4 or TIM-1 inhibited the sensitization and localized allergic responses to OVA. S. aureus is a common commensal organism colonizing the airways, and therefore exposure to superantigens or enterotoxins is a reasonable potential risk factor for allergic sensitization to food proteins. Alternatively, other microbial products derived from colonizing microbes could also potentially act as adjuvants to break normal tolerance responses.

In addition to Th2-promoting costimulatory molecules, the role of regulatory costimulatory molecules have also been investigated. Van Wijk et al. ${ }^{15}$ tested the role of CTLA-4 in tolerance and sensitization to peanut. Although inhibition of CTLA-4 in vivo could enhance oral sensitization to peanut using the adjuvant $\mathrm{CT}$, there was no induction of sensitization when mice were fed peanut in the absence of adjuvant. Therefore, although this molecule could provide a normal brake to the development of sensitization, positive signals were required in addition to the absence of this negative signal for allergic sensitization to occur. All together, these studies suggest that allergic sensitization is a result not only of a loss of tolerance, but due to an active Th2generating immune response driven by DCs. This concept is illustrated in Figure 1.

\section{Manipulation of epithelial barrier}

Another approach to breaking oral tolerance has been to manipulate the normal mucosal barrier. Barrier function is provided not only by the physical presence of tight junctions between epithelial cells, but also by factors such as IgA or digestive enzymes that prevent the uptake of antigenic macromolecules into the body proper. Interference with digestion of food allergens (by antacid treatment of mice) promotes the development of allergic sensitization to digestion-labile allergens such as hazelnut and codfish proteins. ${ }^{40-42}$ This was shown using sucralfate given orally, or ranitidine ( $\mathrm{H} 2$ histamine receptor blocker) or omeprazole (proton pump inhibitor) given systemically. Although sucralfate is aluminum-containing and has adjuvant effects independent of its effects on gastric acid, ${ }^{40}$ the observation that ranitidine or omeprazole could enhance sensitization supports the concept that normal digestive processes are important for the prevention of sensitization. It is unlikely to be due to a simple dose effect, such that more allergen uptake equals more sensitization. However, acid suppression may allow proteins that are normally digestion labile to gain access to the mucosal immune system. 


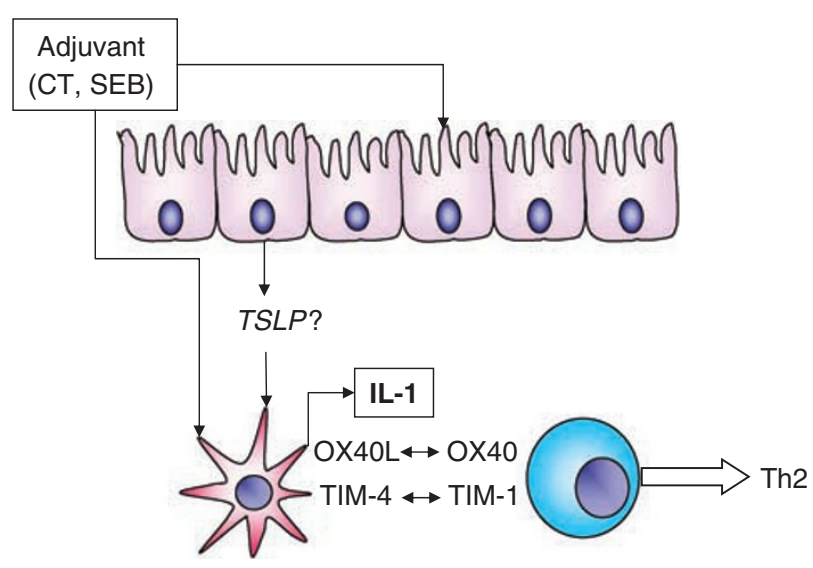

Figure 1 Adjuvants induce Th2 skewing via epithelial cells and dendritic cells. Cholera toxin (CT) and Staphylococcus enterotoxin B (SEB) induce a Th2 skewing response in the gastrointestinal tract when orally administered with antigen. CT induces upregulation of interleukin (IL)-1 within the intestine, and administration of exogenous IL-1 can function in place of CT. It is not yet known if the proallergenic epithelial cytokine thymic stromal lymphopoietin ( TSLP) is also induced by adjuvants. Administration of adjuvants induces maturation of dendritic cells (DCs), including upregulation of OX4OL and TIM-4. Neutralization of these costimulatory molecules has been shown to abrogate Th2 skewing in the gastrointestinal tract.

Another barrier-modulating factor that has been shown to affect sensitization is psychological stress. Modeling of psychological stress in rats through the use of cold restraint or water avoidance leads to decreased barrier function throughout the gastrointestinal tract and increased permeation of macromolecules across enterocytes. ${ }^{43-45}$ Feeding of rats with antigen (plus alum) after water-avoidance stress resulted in sensitization to the fed antigen, whereas feeding nonstressed rats did not result in sensitization. ${ }^{46}$ Outcome measures included antigenspecific IgE production and evidence of intestinal hypersensitivity reactions in response to allergen challenge. Adjuvant was included in these experiments, but manipulation of barrier function was a necessary component for the development of allergic sensitization. Gastrointestinal colonization with the microorganism Candida albicans also was shown to lead to allergic sensitization to a coadministered protein antigen, and this was associated with a decrease in epithelial barrier function (tested by appearance of a fed antigen in the serum). ${ }^{47}$ The authors speculated that the barrier defect was playing a significant role in sensitization, however pathogen-associated molecular patterns associated with C. albicans should not be ruled out as playing a significant adjuvant role.

Hogan and colleagues have recently used a transgenic approach to develop a mouse model of adjuvant-free oral sensitization. ${ }^{48}$ Initial experiments showed that IL-9 was overexpressed in the small intestine of mice with allergen-driven diarrhea and allergic inflammation. Transgenic expression of IL-9 in the intestinal epithelium, using the fatty-acid-binding protein promoter, induced a local mastocytosis, increase in mast-cell degranulation, and increased permeability of the intestinal epithelium. These mice were then susceptible to oral sensitization to OVA in the absence of any exogenous adjuvant.
Sensitization could be inhibited by cromolyn, a mast-cell stabilizer. The authors conclude that the mast-cell-driven changes in epithelial permeability were a critical factor in this susceptibility to allergic sensitization. ${ }^{48}$ In addition, or alternatively, mast-cell degranulation has been shown to induce migration of DCs to the lymph nodes and maturation of those DCs ${ }^{49}$ and mast-cell activators can function as adjuvants. ${ }^{50}$ Thus mast-cell products could be having adjuvant effects in this model independent of antigen uptake across the epithelial barrier.

\section{Immunomodulatory effects of food allergens}

The observation that a relatively conserved group of eight foods is responsible for the vast majority of food allergies despite the variety of the human diet suggests that the common food allergens have inherent properties that make them potent allergens. Shreffler et al. ${ }^{51}$ showed that human DCs treated with peanut extract up-regulate costimulatory molecules, and induce preferential differentiation of naive $\mathrm{T}$ cells into Th2 cytokine-producing cells. The receptor was identified as DC-SIGN, and the protein within the peanut extract identified as Ara h 1. Reports of in vivo responses to peanut support the idea that there may be direct immunomodulatory effects. A single high-dose feed of peanut without adjuvant was reported to induce allergic sensitization in $\mathrm{C} 3 \mathrm{H} / \mathrm{HeJ}$ mice, resulting in anaphylaxis in response to systemic rechallenge 2 weeks after the initial oral exposure. ${ }^{52}$ Anaphylaxis was associated with mast-cell activation (MMCP-1 release), and there was evidence for peanut-specific IgE production. Such high doses of other antigens such as OVA have been associated with deletional tolerance, ${ }^{53}$ but a control protein or food other than peanut was not used to determine if this sensitizing effect was unique to peanut.

\section{Cutaneous sensitization to food allergens}

The skin is a common site of symptoms in patients with food allergy, and a potential explanation for this is that antigenspecific $\mathrm{T}$ cells are first primed at the skin, and subsequently rehomed to the skin. Mice can be sensitized epicutaneously resulting in antigen-specific IgE responses. ${ }^{54-56}$ Sensitization of mice through the skin to OVA, peanut, or hazelnut can induce anaphylactic symptoms when the mice are orally rechallenged. ${ }^{57-59}$ These models do not require adjuvant, although they commonly use mild abrasion of the skin before antigen application. These adjuvant-free animal models support the investigation of the skin as a site of sensitization to food proteins in human food allergy.

\section{EFFECTOR MECHANISMS OF FOOD ALLERGY Antigen uptake}

With the establishment of models of oral sensitization to peanut, studies have begun to emerge on the immunologic mechanisms of anaphylaxis to food allergens. Responsiveness to oral challenge is relatively strain specific, such that $\mathrm{C} 3 \mathrm{H} / \mathrm{HeJ}$ mice are susceptible to oral challenge, whereas $\mathrm{Balb} / \mathrm{c}$ mice and $\mathrm{C} 57 \mathrm{BL} / 6$ mice, although they can be readily sensitized to antigens, require systemic allergen challenge to elicit systemic anaphylaxis, ${ }^{10}$ (Berin and Mayer, unpublished observations). We have 

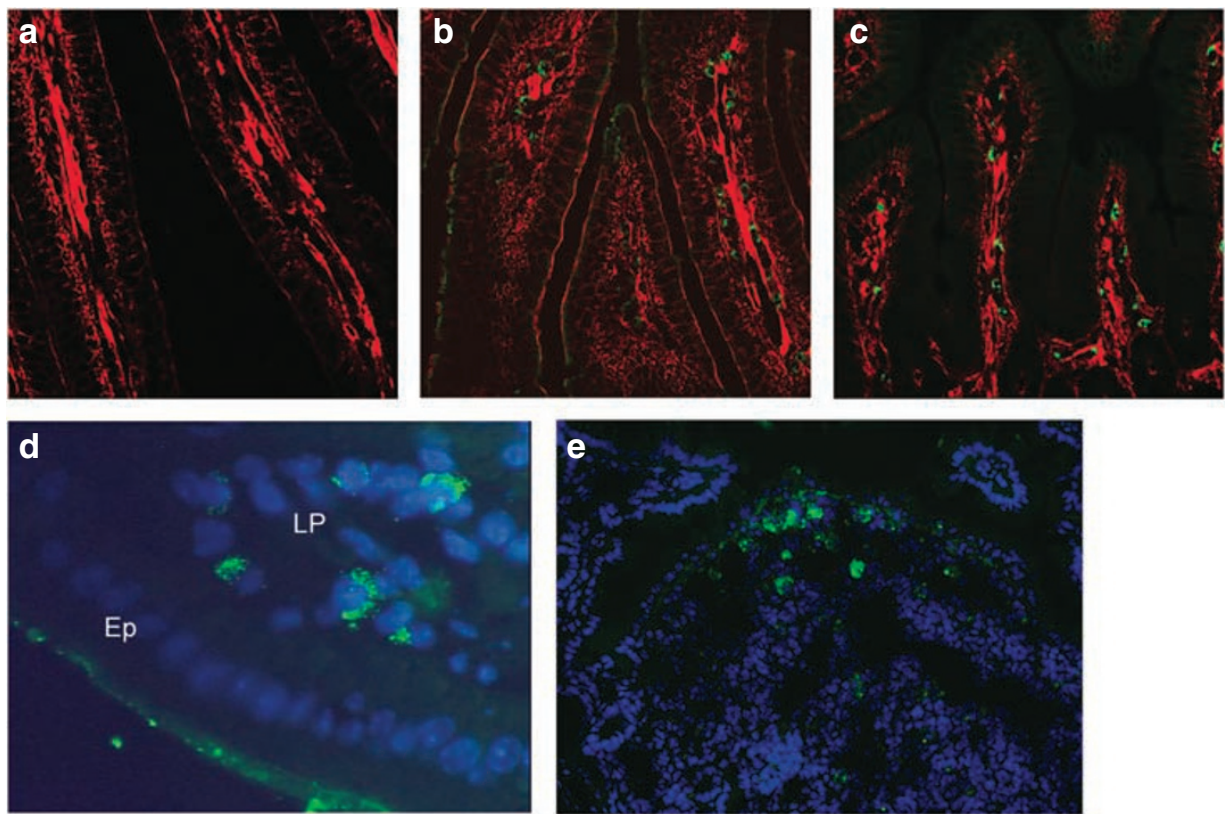

Figure 2 Antigen uptake in the mouse small intestine. Naive $\mathrm{C} 3 \mathrm{H} / \mathrm{HeJ}$ mice were fed fluorescein isothyocyanate (FITC)-labeled milk proteins, and 10-15 min after feeding the intestine was removed, frozen, and cryosections prepared. Sections were counterstained with Texas Red-phalloidin (red, a-c), or 4'-6-diamidino-2-phenylindole (DAPI; blue, d, e). (a) Intestine from unfed mice, showing absence of endogenous green fluorescence. (b-d) At 10 min after feeding, FITC-BLG was clearly present within cells running along the lacteals of the small intestine (b-c). Antigen was cell associated (d) in the lamina propria (LP), and below the level of the epithelium (Ep). Pasteurization of milk protein ( $\alpha$-lactalbumin, e) rendered the soluble milk proteins insoluble, and the particulate proteins were preferentially taken up the Peyer's patch.

shown that the susceptibility of the $\mathrm{C} 3 \mathrm{H}$ strain to anaphylaxis is not TLR4 dependent, ${ }^{10}$ although other investigators have reported a TLR4 dependence of sensitization and anaphylaxis to peanut. ${ }^{13}$

To elicit anaphylaxis after oral ingestion, allergen must first breach the epithelial barrier to reach either local or distal effector cells such as mast cells or basophils. We studied the response of $\mathrm{C} 3 \mathrm{H} / \mathrm{HeJ}$ mice to three major milk allergens: casein, $\beta$-lactoglobulin, and $\alpha$-lactalbumin. ${ }^{60}$ Casein, which is normally found contained in micelle-like structures, was significantly better at inducing allergic sensitization (as measured by allergen-specific immunoglobulin and cytokine production) than the soluble antigens $\beta$-lactoglobulin or $\alpha$-lactalbumin. Despite this, the soluble antigens could elicit anaphylaxis when delivered orally, whereas casein required systemic delivery to induce anaphylaxis. Investigation of the uptake of these proteins showed that the soluble antigens readily passed the epithelial barrier and could be detected in the lamina propria of the small intestine (see Figure 2). In contrast, casein could not bypass the absorptive epithelium but was detected in the Peyer's patches. Pasteurization of the soluble antigens rendered them particulate, and like casein, they were very effective at sensitization but could no longer trigger anaphylaxis when delivered orally ${ }^{60}$ Selective Peyer's patch uptake has also been described for peanut proteins. ${ }^{61}$ Thus Peyer's patch trafficking was associated with increased sensitization whereas efficient villous trafficking was associated with oral antigen-induced anaphylaxis. Therefore both the level of sensitization and degree of uptake across absorptive epithelium appear to be important factors contributing to the likelihood of anaphylaxis to luminal food allergens.

\section{IgE-mediated antigen sampling across the intestinal epithelium}

Ingestion of food allergens by food-allergic individuals leads to symptoms that can occur very rapidly after ingestion. It has not been possible to pinpoint the site of antigen absorption: it is possible that some uptake of antigen is occurring in the mouth across buccal or sublingual mucosa. The small intestine, in particular the jejunum, is thought to be the site of greatest absorption in the gastrointestinal tract. In vitro challenge of jejunum from sensitized animals has shown that luminal antigen challenge leads to detectable local hypersensitivity responses within a few minutes (3-5 min). ${ }^{62-64}$ To understand how antigen could trigger these responses so quickly, electron microscopy analysis of antigen uptake was performed comparing jejunum from naive vs. sensitized animals. ${ }^{65,66}$ Sensitization induced greater antigen uptake via a transcellular uptake pathway. However, this was not observed using a bystander antigen, indicating that there was specificity or recognition in this uptake process. ${ }^{66}$ That specificity was found to be due to IgE-mediated antigen uptake through the low-affinity IgE receptor $\mathrm{CD} 23$ that was expressed on intestinal epithelial cells. ${ }^{67,68}$ We have shown that the CD23a isoform is constitutively expressed by primary human intestinal epithelial cells, and can be detected in the stool of patients with food allergy but not normal subjects, ${ }^{69}$ We and others have shown that CD23 functions as a bidirectional transporter of IgE in vitro using human intestinal epithelial cell lines ${ }^{69-71}$ 


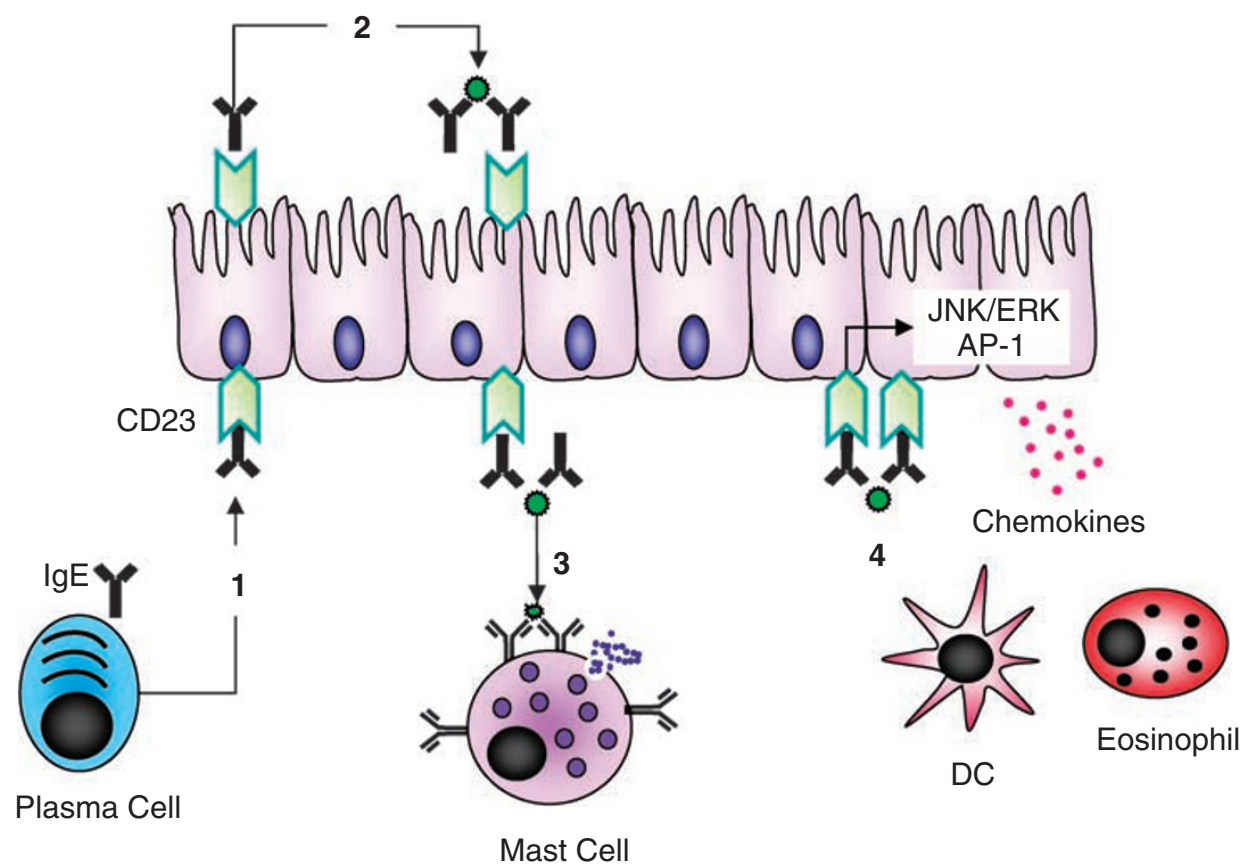

Figure 3 Functions of epithelial CD23 in the allergic gastrointestinal tract. (1) CD23 functions as a bidirectional transporter of immunoglobulin-E ( $\mathrm{lgE}$ ), transporting antigen from the intestine into the lumen, where it can (2) bind antigen, form complexes and be transported back in an apical-tobasolateral direction across the epithelial cell. After transport, intact complexes can (3) trigger mast cells to induce local hypersensitivity reactions, or alternatively (4) act back on basal CD23 to trigger pro-inflammatory epithelial signaling and release of chemokines (interleukin (IL)-8 and CCL20). These chemokines may then participate in recruitment of inflammatory cells as part of a late-phase reaction, and recruitment of dendritic cells to amplify the adaptive immune response via antigen capture and presentation.

and in addition CD23 can sample antigen-IgE complexes from the luminal side for delivery to subepithelial immune cells. ${ }^{69,72}$ The in vivo consequences of this antigen sampling system are not yet fully understood. We know that soluble antigens can penetrate across the intestinal epithelial barrier in the absence of this sampling mechanism, but IgE-CD23 may help antigens that traffic poorly (i.e., casein, peanut, or other particulate antigens) to traverse the epithelium. In addition, we have recently shown that ligation of $\mathrm{CD} 23$ by IgE-antigen complexes can lead to epithelial chemokine release, including CCL20, and induce the recruitment of DCs. ${ }^{73}$ Thus, CD23 may function to bring small quantities of antigen across the epithelium for delivery to recruited DCs, and thereby function as a selective conduit between the lumen and the mucosal immune system. This is illustrated in Figure 3.

\section{Mechanisms of systemic anaphylaxis}

Breaching the epithelial barrier is the first step in an allergic response to food proteins. The most severe responses to allergen in mouse models include systemic anaphylaxis characterized by cardiovascular compromise, respiratory distress, and hypothermia. There have been two main pathways of anaphylaxis described in mice. ${ }^{74}$ The classic pathway is activated by cross-linking of IgE bound to high affinity FceRI receptors on mast cells, leading to release of an array of mediators including histamine, platelet activating factor (PAF), leukotrienes, and proteases. Blockade of histamine can prevent the hypothermia triggered by this pathway of anaphylaxis. ${ }^{75}$ The alternative pathway is activated by cross-linking IgG bound to Fc $\gamma$ RIII on macrophages, and the symptoms are triggered by $\mathrm{PAF}^{76}$ Dose of antigen is a critical factor in the induction of the different pathways of anaphylaxis, and small doses of antigen have been shown to favor the classic pathway, whereas large doses are required for triggering the alternative pathway. ${ }^{77}$ However, in most animal models of food allergy there is the generation of both antigenspecific IgE and IgG, and IgG may then function to inhibit activation of the classic pathway by competition for the antigen. ${ }^{77}$ It should be noted that only a very small fraction of ingested antigen is thought to be absorbed across the intestinal barrier in an immunologically intact form, so that the dose of antigen available systemically would be predicted to be very small. For example, feeding of animals with antigen doses in the milligram range leads to peak portal vein or lymph concentrations in the $\mathrm{ng} / \mathrm{ml}$ range (to a high range of $1 \mu \mathrm{g} / \mathrm{ml}$ ). ${ }^{78}$ This low antigen availability may limit the potential role of IgG-mediated anaphylaxis to food, and therefore studies utilizing systemic antigen challenge should be interpreted with some caution.

The dependence on $\mathrm{C} 3 \mathrm{H}$ mice in food allergy models greatly limits the availability of genetically modified mice to test the role of immune pathways in food-induced anaphylaxis. However, the use of other strains such as C57BL/6 mice requires the use of systemic allergen challenge. Sun et al. modified a CT-induced model of peanut allergy for use in C57BL/6 mice, such that systemic rechallenge with a relatively high dose of peanut antigen $(5 \mathrm{mg})$ after oral sensitization with peanut butter and CT leads to systemic anaphylaxis associated with a drop in body temperature, release of histamine and leukotrienes into the peritoneal cavity, and loss of albumin into the peritoneal cavity. ${ }^{14}$ 
A late-phase response was observed consisting of an inflammatory infiltrate into the peritoneal cavity. Anaphylaxis was completely abolished in B-cell-deficient or CD40L-deficient mice, despite normal cellular (late-phase) responses. In addition, anaphylaxis was completely abolished in mast-cell-deficient $\left(\mathrm{W} / \mathrm{W}^{\mathrm{v}}\right)$ mice, arguing against a role for the alternative pathway of anaphylaxis in this model. ${ }^{14}$ Interestingly, anaphylaxis was only partially reversed in FceRI-knockout mice, showing that this peanut-induced anaphylaxis does not strictly conform to the classic anaphylaxis pathway. Basophils have recently been reported to mediate IgG1-induced anaphylaxis through the release of PAF in mice, ${ }^{79}$ demonstrating yet another pathway of anaphylaxis in addition to the two previously described. The role of basophils in food allergy models has not yet been tested. These studies on the relative role of IgE vs. other isotypes should be confirmed in other mouse strains, using oral challenge where possible or minimizing the allergen challenge dose to more carefully mimic oral antigen exposure. Studies such as these may help to solve the current controversy regarding the relative role of histamine vs. PAF in human food-induced anaphylaxis.

An interesting nonimmunologic or anaphylactoid effect of peanut has been described preliminarily by Finkelman and colleagues. ${ }^{74}$ Systemic challenge with peanut could induce anaphylaxis in naive animals, in a macrophage- and complement-dependent manner. The relevance of anaphylactoid-type mechanisms to human peanut-induced anaphylaxis is unknown, but this direct activation of immune cells could potentially contribute to an adjuvant-type effect of peanut.

\section{Gastrointestinal manifestations of food allergy}

Although mouse models of oral antigen-induced systemic anaphylaxis have been difficult to develop, a number of groups have studied local allergic manifestations in the intestine in response to food allergens. Two main approaches have been taken, both starting with systemic sensitization of rats or mice with antigen plus adjuvant. The first method uses an in vitro approach to study hypersensitivity reactions in the intestine. The second approach uses repetitive oral challenge to induce allergic inflammation and diarrhea. The first approach uses intestinal segments removed from sensitized mice or rats, mounted in Ussing chambers, and challenged in vitro with antigen delivered to the luminal or the serosal surface of the tissue. The tissue is voltage clamped, allowing for the measurement of net active ion transport across the tissue. These studies showed that antigen challenge of tissue from sensitized animals induced a rapid increase in current across the tissue that was driven by active chloride secretion by intestinal epithelial cells. ${ }^{62,63,80}$ In addition, in vitro challenge led to a decrease in epithelial barrier function associated with permeation of macromolecules across the epithelial tight junctions. ${ }^{66}$ The use of mast-cell-deficient $\mathrm{W} / \mathrm{W}^{\mathrm{v}}$ mice and $\mathrm{Ws} / \mathrm{Ws}$ rats demonstrated that these changes in ion secretion and epithelial permeability were mast-cell dependent. ${ }^{64,65}$ Ion secretion responses could be passively transferred with serum, and this was abolished with heat treatment of the serum, consistent with a role for IgE or other heat-labile molecules. ${ }^{80}$ Furthermore, pharmacologic blockade of histamine, serotonin, and prostaglandins in combination suppressed the ion transport response to antigen challenge. ${ }^{62}$

Increased epithelial ion secretion and decreased barrier function were thought to be driving forces for antigen-driven diarrhea, but despite the electrophysiologic results, a single oral challenge did not lead to diarrheal responses. Brandt et al. ${ }^{81}$ used repetitive oral antigen challenges in systemically sensitized mice to induce allergic diarrhea in vivo (alum/OVA model). In addition, they observed mastocytosis and cytokine changes in the small intestine that were consistent with gastrointestinal changes described in human food allergic subjects after allergen exposure. ${ }^{82}$ They were then able to confirm the IgE- and mast-cell-dependence of the diarrhea in vivo. ${ }^{81}$ In contrast to the findings in vitro, histamine was not found to be a major contributing mediator of allergic diarrhea. A combination of PAF and serotonin blockade was able to abrogate the onset of symptoms in response to allergen challenge, although inhibition of neither alone could prevent diarrhea.

In addition to mast cells and IgE, T lymphocytes have also been shown to play a critical role in the development of allergic diarrhea. Kweon et al. ${ }^{83}$ developed a model of allergic diarrhea affecting the large intestine by systemic sensitization with OVA in complete Freund's adjuvant (CFA/OVA model) followed by repetitive feeding with OVA. They found that transfer of CD4+ $\mathrm{T}$ cells from the spleen of sensitized mice to SCID mice could transfer allergen-induced diarrhea. In addition, the role of $\mathrm{T}$ cells was highlighted by showing that treatment of mice with FTY720, a sphingosine-1-receptor agonist that sequesters $\mathrm{T}$ cells in lymph nodes, prevents allergic diarrhea in the CFA/OVA model. ${ }^{84} \mathrm{We}$ recently showed that in the alum/OVA model, CD4 $+\mathrm{T}$ cells activated in the draining mesenteric lymph node by oral OVA challenge could transfer disease to naive and immunologically intact $\mathrm{Balb} / \mathrm{c}$ mice. ${ }^{85}$ As this model has previously been shown to be mast-cell- and IgE-dependent, the communication between $\mathrm{T}$ cells and mast cells needs to be investigated to determine at what point in disease pathogenesis $\mathrm{T}$ cells can play a role. Studies from Forbes et al..$^{48}$ suggest that T-cell-derived IL-9 may be a critical factor in $\mathrm{T}$ cell/mast cell interactions. As outlined above, IL-9 knockout mice do not get gastrointestinal manifestations of food allergy, and overexpression of IL-9 can replicate the allergen-driven diarrhea model. Therefore, a single T-cell cytokine can orchestrate allergic inflammation in the gut.

Hino et al. ${ }^{86}$ used the CFA/OVA model to show that despite the STAT- 6 dependence of the model and local overexpression of Th2 cytokines, IL-12 p40 was critical to the development of allergic diarrhea. Further analysis showed that the IL-12 that was expressed in the large intestine was in the form of IL-12 p80 (p40 homodimers). The downstream targets of the p40 homodimers were not assessed. In contrast to p80, treatment of mice with exogenous IL-12 p70 prevents allergic diarrhea in the CFA/OVA model. ${ }^{87}$ Thus the balance of IL-12 subunits or family members appears to be critical for the development of symptoms in this model.

One very interesting question regarding the CFA/OVA model and the alum/OVA model is why the allergic inflammation in the CFA/OVA model is localized to the large intestine whereas 


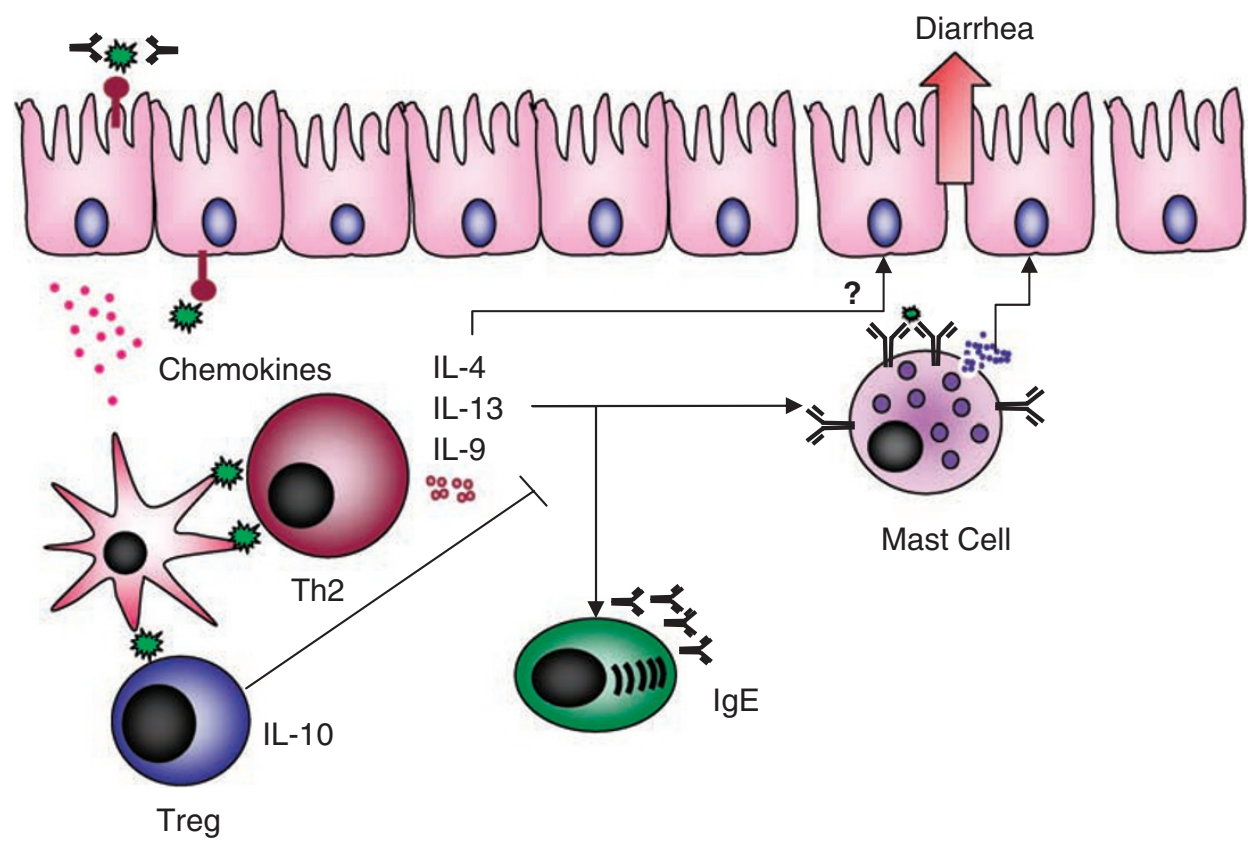

Figure 4 Immunophysiology of gastrointestinal manifestations of food allergy. Oral antigen challenge of sensitized mice leads to a Th2 inflammation and allergen-induced diarrhea. The diarrhea symptoms are driven by changes in epithelial ion transport and permeability that are induced by mastcell degranulation via cross-linking of immunoglobulin-E (IgE) bound to Fc\&RI on the cell surface. Th2 lymphocytes are critical in the development of allergic diarrhea through the release of the cytokines interleukin (IL)-4, IL-13, and IL-9. These cytokines can promote mastocytosis and IgE class switching, but it is not known if there are also direct effects of the Th2 lymphocytes on gastrointestinal physiology in allergic disease. IL-10 producing CD4 + CD25 + T regulatory cells (Treg) are also induced by oral antigen challenge, and these cells provide inhibitory signals preventing allergic disease. Epithelial cells function not only as an antigen-sampling cell, but also as a source of chemokines that promote the allergic inflammation.

the inflammation in the alum/OVA model is in the small intestine. One clue to this question comes from a study by Takayama et al. ${ }^{88}$ that used gestational treatment with anti-IL-7R $\alpha$ antibody to abolish Peyer's patches in offspring mice. They observed that mice without Peyer's patches became more susceptible to CFA/ OVA allergic diarrhea, and that the Peyer's patches were sites of antigen-specific IL-10 production. Transfer of CD $4+\mathrm{CD} 25+$ cells from the Peyer's patch prevented diarrhea development in Peyer's patch-null mice. Alternatively, neutralization of IL-10 or removal of CD25 + cells from PP-intact mice resulted in diarrhea. ${ }^{88}$ Therefore, the authors concluded that regulatory cells are generated in the Peyer's patch that prevent the development of allergic inflammation in the small intestine. These studies show that even in a pathogenic disease model, there is a tolerance response that is generated by antigen exposure via the gastrointestinal tract. Figure 4 illustrates the immunologic mechanisms of gastrointestinal manifestations of food allergy.

\section{SUMMARY}

Animal models and in vitro systems of food allergy have highlighted several critical factors promoting sensitization to food allergens. Factors that perturb the epithelial barrier, as well as innate characteristics of the antigen such as endogenous adjuvant activity (for peanut) or particulate form (casein) promote allergic sensitization. Microbial products function as adjuvants for allergic sensitization by altering the phenotype of mucosal DCs and promoting the expression of Th2-stimulating costimulatory molecules. Studies need to be performed to determine if there are endogenous, environmental, or dietary factors that can promote sensitization by these pathways. Further studies need to be performed on mechanisms of systemic anaphylaxis in mouse models of food allergy, with particular focus on dissecting mechanisms of anaphylaxis at physiologic doses of food allergens.

\section{ACKNOWLEDGMENTS}

This work was supported by funding from the National Institutes of Health (Al044236 to MCB; Al044236, DK072201, Al04343, Al061093, and Al066738 to LM) and the Food Allergy Initiative (to MCB).

\section{DISCLOSURE}

The authors declared no conflict of interest.

C) 2009 Society for Mucosal Immunology

\section{REFERENCES}

1. Chehade, M. \& Mayer, L. Oral tolerance and its relation to food hypersensitivities. J. Allergy Clin. Immunol. 115, 3-12; quiz 3 (2005).

2. Kraus, T.A., Toy, L., Chan, L., Childs, J. \& Mayer, L. Failure to induce oral tolerance to a soluble protein in patients with inflammatory bowel disease. Gastroenterology 126, 1771-1778 (2004).

3. Elson, C.O. \& Ealding, W. Cholera toxin feeding did not induce oral tolerance in mice and abrogated oral tolerance to an unrelated protein antigen. J. Immunol. 133, 2892-2897 (1984).

4. Snider, D.P., Marshall, J.S., Perdue, M.H. \& Liang, H. Production of IgE antibody and allergic sensitization of intestinal and peripheral tissues after oral immunization with protein Ag and cholera toxin. J. Immunol. 153, 647-657 (1994).

5. Li, X.M. et al. A murine model of peanut anaphylaxis: T- and B-cell responses to a major peanut allergen mimic human responses. J. Allergy Clin. Immunol. 106, 150-8 (2000). 
6. Li, X.M., Schofield, B.H., Huang, C.K., Kleiner, G.I. \& Sampson, H.A. A murine model of IgE-mediated cow's milk hypersensitivity. J. Allergy Clin. Immunol. 103, 206-214 (1999).

7. Lee, S.Y. et al. Murine model of buckwheat allergy by intragastric sensitization with fresh buckwheat flour extract. J. Korean Med. Sci. 20, 566-572 (2005).

8. Foss, N., Duranti, M., Magni, C. \& Frokiaer, H. Assessment of lupin allergenicity in the cholera toxin model: induction of lgE response depends on the intrinsic properties of the conglutins and matrix effects. Int. Arch. Allergy Immunol. 141, 141-150 (2006).

9. Adel-Patient, K., Bernard, H., Ah-Leung, S., Creminon, C. \& Wal, J.M. Peanut- and cow's milk-specific lgE, Th2 cells and local anaphylactic reaction are induced in Balb/c mice orally sensitized with cholera toxin. Allergy 60, 658-664 (2005).

10. Berin, M.C., Zheng, Y., Domaradzki, M., Li, X.M. \& Sampson, H.A. Role of TLR4 in allergic sensitization to food proteins in mice. Allergy 61, 64-71 (2006).

11. Frossard, C.P., Hauser, C. \& Eigenmann, P.A. Oral carrageenan induces antigen-dependent oral tolerance: prevention of anaphylaxis and induction of lymphocyte anergy in a murine model of food allergy. Pediatr. Res. 49, 417-422 (2001).

12. Bashir, M.E., Andersen, P., Fuss, I.J., Shi, H.N. \& Nagler-Anderson, C. An enteric helminth infection protects against an allergic response to dietary antigen. J. Immunol. 169, 3284-92 (2002).

13. Bashir, M.E., Louie, S., Shi, H.N. \& Nagler-Anderson, C. Toll-like receptor 4 signaling by intestinal microbes influences susceptibility to food allergy. J. Immunol. 172, 6978-6987 (2004).

14. Sun, J. et al. Impact of CD40 ligand, B cells, and mast cells in peanutinduced anaphylactic responses. J. Immunol. 179, 6696-6703 (2007).

15. van Wijk, F. et al. CTLA-4 signaling regulates the intensity of hypersensitivity responses to food antigens, but is not decisive in the induction of sensitization. J. Immunol. 174, 174-179 (2005).

16. Yamamoto, M. et al. Enterotoxin adjuvants have direct effects on T cells and antigen-presenting cells that result in either interleukin-4-dependent or -independent immune responses. J. Infect. Dis. 182, 180-190 (2000).

17. Gagliardi, M.C., Sallusto, F., Marinaro, M., Langenkamp, A., Lanzavecchia, A. \& De Magistris, M.T. Cholera toxin induces maturation of human dendritic cells and licences them for Th2 priming. Eur. J. Immunol. 30, 2394-2403 (2000)

18. Williamson, E., Westrich, G.M. \& Viney, J.L. Modulating dendritic cells to optimize mucosal immunization protocols. J. Immunol. 163, 3668-3675 (1999).

19. Staats, H.F. \& Ennis, F.A. Jr IL-1 is an effective adjuvant for mucosal and systemic immune responses when coadministered with protein immunogens. J. Immunol. 162, 6141-6147 (1999).

20. Bromander, A., Holmgren, J. \& Lycke, N. Cholera toxin stimulates IL-1 production and enhances antigen presentation by macrophages in vitro. J. Immunol. 146, 2908-2914 (1991).

21. Eisenbarth, S.C., Colegio, O.R., O'Connor, W., Sutterwala, F.S. \& Flavell, R.A. Crucial role for the Nalp3 inflammasome in the immunostimulatory properties of aluminium adjuvants. Nature 453, 1122-1126 (2008).

22. Kool, M. et al. Cutting Edge: alum adjuvant stimulates inflammatory dendritic cells through activation of the NALP3 inflammasome. J. Immunol. 181, 3755-3759 (2008).

23. Huang, F.P. et al. A discrete subpopulation of dendritic cells transports apoptotic intestinal epithelial cells to T cell areas of mesenteric lymph nodes. J. Exp. Med. 191, 435-444 (2000).

24. Liu, L.M. \& MacPherson, G.G. Antigen acquisition by dendritic cells: intestinal dendritic cells acquire antigen administered orally and can prime naive T cells in vivo. J. Exp. Med. 177, 1299-1307 (1993).

25. Niess, J.H. et al. CX3CR1-mediated dendritic cell access to the intestinal lumen and bacterial clearance. Science 307, 254-258 (2005).

26. Rescigno, M. et al. Dendritic cells express tight junction proteins and penetrate gut epithelial monolayers to sample bacteria. Nat. Immunol. 2, 361-367 (2001).

27. Blazquez, A.B. \& Berin, M.C. Gastrointestinal dendritic cells promote Th2 skewing via OX4OL. J. Immunol. 180, 4441-4450 (2008).

28. Anjuere, F. et al. In vivo adjuvant-induced mobilization and maturation of gut dendritic cells after oral administration of cholera toxin. J. Immunol. 173, 5103-5111 (2004).

29. Anosova, N.G., Chabot, S., Shreedhar, V., Borawski, J.A., Dickinson, B.L. \& Neutra, M.R. Cholera toxin, E. coli heat-labile toxin, and non-toxic derivatives induce dendritic cell migration into the follicle-associated epithelium of Peyer"s patches. Mucosal Immunology 1, 59-67 (2008).

30. Shreedhar, V.K., Kelsall, B.L. \& Neutra, M.R. Cholera toxin induces migration of dendritic cells from the subepithelial dome region to T- and B-cell areas of Peyer's patches. Infect. Immun. 71, 504-509 (2003).

31. Ekkens, M.J. et al. The role of OX40 ligand interactions in the development of the Th2 response to the gastrointestinal nematode parasite Heligmosomoides polygyrus. J. Immunol. 170, 384-393 (2003).

32. Hoshino, A. et al. Critical role for OX40 ligand in the development of pathogenic Th2 cells in a murine model of asthma. Eur. J. Immunol. 33, 861-869 (2003).

33. Ishii, N., Ndhlovu, L.C., Murata, K., Sato, T., Kamanaka, M. \& Sugamura, K. OX40 (CD134) and OX40 ligand interaction plays an adjuvant role during in vivo Th2 responses. Eur. J. Immunol. 33, 2372-2381 (2003).

34. Ito, T., Wang, Y.H., Duramad, O., Hori, T., Delespesse, G.J. \& Watanabe, N. et al. TSLP-activated dendritic cells induce an inflammatory Thelper type 2 cell response through OX4O ligand. J. Exp. Med. 202, 1213-1223 (2005).

35. Salek-Ardakani, S. et al. OX40 (CD134) controls memory Thelper 2 cells that drive lung inflammation. J. Exp. Med. 198, 315-324 (2003).

36. Amsen, D., Blander, J.M., Lee, G.R., Tanigaki, K., Honjo, T. \& Flavell, R.A. Instruction of distinct CD4 Thelper cell fates by different notch ligands on antigen-presenting cells. Cell 117, 515-526 (2004).

37. Krawczyk, C.M., Sun, J. \& Pearce, E.J. Th2 differentiation is unaffected by Jagged2 expression on dendritic cells. J. Immunol. 180, 7931-7937 (2008).

38. Feng, B.S. et al. Disruption of T-cell immunoglobulin and mucin domain molecule (TIM)-1/TIM4 interaction as a therapeutic strategy in a dendritic cell-induced peanut allergy model. J. Allergy Clin. Immunol. 122, 55-61 e1-7 (2008)

39. Yang, P.C. et al. TIM-4 expressed by mucosal dendritic cells plays a critical role in food antigen-specific Th2 differentiation and intestinal allergy. Gastroenterology 133, 1522-1533 (2007).

40. Brunner, R. et al. The impact of aluminium in acid-suppressing drugs on the immune response of BALB/c mice. Clin. Exp. Allergy 37, 1566-1573 (2007).

41. Scholl, I. et al. Antiulcer drugs promote oral sensitization and hypersensitivity to hazelnut allergens in BALB/c mice and humans. Am. J. Clin. Nutr. 81, 154-160 (2005).

42. Untersmayr, E. et al. Antacid medication inhibits digestion of dietary proteins and causes food allergy: a fish allergy model in BALB/c mice. J. Allergy Clin. Immunol. 112, 616-623 (2003).

43. Santos, J. et al. Corticotropin-releasing hormone mimics stress-induced colonic epithelial pathophysiology in the rat. Am. J. Physiol. 277, G391G399 (1999).

44. Kiliaan, A.J. et al. Stress stimulates transepithelial macromolecular uptake in rat jejunum. Am. J. Physiol. 275, G1037-G1044 (1998).

45. Saunders, P.R., Kosecka, U., McKay, D.M. \& Perdue, M.H. Acute stressors stimulate ion secretion and increase epithelial permeability in rat intestine. Am. J. Physiol. 267, G794-G799 (1994).

46. Yang, P.C., Jury, J., Soderholm, J.D., Sherman, P.M., McKay, D.M. \& Perdue, M.H. Chronic psychological stress in rats induces intestinal sensitization to luminal antigens. Am. J. Pathol. 168, 104-114 quiz 363 (2006).

47. Yamaguchi, N. et al. Gastrointestinal Candida colonisation promotes sensitisation against food antigens by affecting the mucosal barrier in mice. Gut 55, 954-960 (2006).

48. Forbes, E.E. et al. IL-9- and mast cell-mediated intestinal permeability predisposes to oral antigen hypersensitivity. J. Exp. Med. 205, 897-913 (2008).

49. Jawdat, D.M., Albert, E.J., Rowden, G., Haidl, I.D. \& Marshall, J.S. IgEmediated mast cell activation induces Langerhans cell migration in vivo. J. Immunol. 173, 5275-5282 (2004).

50. McLachlan, J.B. et al. Mast cell activators: a new class of highly effective vaccine adjuvants. Nat. Med. 14, 536-541 (2008).

51. Shreffler, W.G. et al. The major glycoprotein allergen from Arachis hypogaea, Ara h 1, is a ligand of dendritic cell-specific ICAM-grabbing nonintegrin and acts as a Th2 adjuvant in vitro. J. Immunol. 177, 3677-3685 (2006).

52. Proust, B. et al. A single oral sensitization to peanut without adjuvant leads to anaphylaxis in mice. Int. Arch. Allergy Immunol. 146, 212-218 (2008).

53. Chen, Y., Inobe, J., Marks, R., Gonnella, P., Kuchroo, V.K. \& Weiner, H.L. Peripheral deletion of antigen-reactive T cells in oral tolerance. Nature 376, 177-180 (1995). 
54. Herrick, C.A., MacLeod, H., Glusac, E., Tigelaar, R.E. \& Bottomly, K. Th2 responses induced by epicutaneous or inhalational protein exposure are differentially dependent on IL-4. J. Clin. Invest. 105, 765-775 (2000).

55. Spergel, J.M., Mizoguchi, E., Brewer, J.P., Martin, T.R., Bhan, A.K. \& Geha, R.S. Epicutaneous sensitization with protein antigen induces localized allergic dermatitis and hyperresponsiveness to methacholine after single exposure to aerosolized antigen in mice. J. Clin. Invest. 101, 1614-1622 (1998).

56. Wang, L.F., Lin, J.Y., Hsieh, K.H. \& Lin, R.H. Epicutaneous exposure of protein antigen induces a predominant Th2-like response with high lgE production in mice. J. Immunol. 156, 4077-4082 (1996).

57. Strid, J., Hourihane, J., Kimber, I., Callard, R. \& Strobel, S. Epicutaneous exposure to peanut protein prevents oral tolerance and enhances allergic sensitization. Clin. Exp. Allergy 35, 757-766 (2005).

58. Hsieh, K.Y., Tsai, C.C., Wu, C.H. \& Lin, R.H. Epicutaneous exposure to protein antigen and food allergy. Clin. Exp. Allergy 33, 1067-1075 (2003).

59. Birmingham, N.P. et al. An adjuvant-free mouse model of tree nut allergy using hazelnut as a model tree nut. Int. Arch. Allergy Immunol. 144, 203-210 (2007).

60. Roth-Walter, F. et al. Pasteurization of milk proteins promotes allergic sensitization by enhancing uptake through Peyer's patches. Allergy 63, 882-890 (2008).

61. Chambers, S.J., Wickham, M.S., Regoli, M., Bertelli, E., Gunning, P.A. \& Nicoletti, C. Rapid in vivo transport of proteins from digested allergen across pre-sensitized gut. Biochem. Biophys. Res. Commun. 325, 1258-1263 (2004).

62. Crowe, S.E., Sestini, P. \& Perdue, M.H. Allergic reactions of rat jejunal mucosa. Ion transport responses to luminal antigen and inflammatory mediators. Gastroenterology 99, 74-82 (1990).

63. Perdue, M.H. \& Gall, D.G. Intestinal anaphylaxis in the rat: jejunal response to in vitro antigen exposure. Am. J. Physiol. 250, G427-G431 (1986).

64. Perdue, M.H., Masson, S., Wershil, B.K. \& Galli, S.J. Role of mast cells in ion transport abnormalities associated with intestinal anaphylaxis. Correction of the diminished secretory response in genetically mast celldeficient WNV mice by bone marrow transplantation. J. Clin. Invest. 87, 687-693 (1991).

65. Berin, M.C., Kiliaan, A.J., Yang, P.C., Groot, J.A., Kitamura, Y. \& Perdue, M.H. The influence of mast cells on pathways of transepithelial antigen transport in rat intestine. J. Immunol. 161, 2561-2566 (1998).

66. Berin, M.C., Kiliaan, A.J., Yang, P.C., Groot, J.A., Taminiau, J.A. \& Perdue, M.H. Rapid transepithelial antigen transport in rat jejunum: impact of sensitization and the hypersensitivity reaction. Gastroenterology 113, 856-864 (1997).

67. Yang, P.C., Berin, M.C., Yu, L.C., Conrad, D.H. \& Perdue, M.H. Enhanced intestinal transepithelial antigen transport in allergic rats is mediated by $\lg \mathrm{E}$ and CD23 (FcepsilonRIII). J. Clin. Invest. 106, 879-886 (2000).

68. Yu, L.C. et al. Enhanced transepithelial antigen transport in intestine of allergic mice is mediated by lgE/CD23 and regulated by interleukin-4. Gastroenterology 121, 370-381 (2001).

69. Li, H. et al. Transcytosis of IgE-antigen complexes by CD23a in human intestinal epithelial cells and its role in food allergy. Gastroenterology 131, 47-58 (2006).

70. Montagnac, G. et al. Intracellular trafficking of CD23: differential regulation in humans and mice by both extracellular and intracellular exons. J. Immunol. 174, 5562-5572 (2005).
71. Tu, Y. et al. CD23-mediated lgE transport across human intestinal epithelium: inhibition by blocking sites of translation or binding. Gastroenterology 129, 928-940 (2005).

72. Tu, Y. \& Perdue, M.H. CD23-mediated transport of lgE/immune complexes across human intestinal epithelium: role of p38 MAPK. Am. J. Physiol. Gastrointest. Liver Physiol. 291, G532-G538 (2006).

73. Li, H., Chehade, M., Liu, W., Xiong, H., Mayer, L. \& Berin, M.C. AllergenIgE complexes trigger CD23-dependent CCL20 release from human intestinal epithelial cells. Gastroenterology 133, 1905-1915 (2007).

74. Finkelman, F.D. Anaphylaxis: lessons from mouse models. J. Allergy Clin. Immunol. 120, 506-515 quiz 16-7 (2007).

75. Makabe-Kobayashi, Y. et al. The control effect of histamine on body temperature and respiratory function in lgE-dependent systemic anaphylaxis. J. Allergy Clin. Immunol. 110, 298-303 (2002).

76. Strait, R.T., Morris, S.C., Yang, M., Qu, X.W. \& Finkelman, F.D. Pathways of anaphylaxis in the mouse. J. Allergy Clin. Immunol. 109, 658-668 (2002).

77. Strait, R.T., Morris, S.C. \& Finkelman, F.D. IgG-blocking antibodies inhibit IgE-mediated anaphylaxis in vivo through both antigen interception and Fc gamma Rllb cross-linking. J. Clin. Invest. 116, 833-841 (2006).

78. Warshaw, A.L., Walker, W.A., Cornell, R. \& Isselbacher, K.J. Small intestinal permeability to macromolecules. Transmission of horseradish peroxidase into mesenteric lymph and portal blood. Lab. Invest. 25, 675-684 (1971).

79. Tsujimura, Y. et al. Basophils play a pivotal role in immunoglobulin-Gmediated but not immunoglobulin-E-mediated systemic anaphylaxis. Immunity 28, 581-589 (2008).

80. Kosecka, U., Marshall, J.S., Crowe, S.E., Bienenstock, J. \& Perdue, M.H. Pertussis toxin stimulates hypersensitivity and enhances nerve-mediated antigen uptake in rat intestine. Am. J. Physiol. 267, G745-G753 (1994).

81. Brandt, E.B. et al. Mast cells are required for experimental oral allergeninduced diarrhea. J. Clin. Invest. 112, 1666-1677 (2003).

82. Lin, X.P. et al. Local allergic reaction in food-hypersensitive adults despite a lack of systemic food-specific IgE. J. Allergy Clin. Immunol. 109, 879-887 (2002).

83. Kweon, M.N., Yamamoto, M., Kajiki, M., Takahashi, I. \& Kiyono, H. Systemically derived large intestinal CD4(+) Th2 cells play a central role in STAT6-mediated allergic diarrhea. J. Clin. Invest. 106, 199-206 (2000).

84. Kurashima, Y. et al. Sphingosine 1-phosphate-mediated trafficking of pathogenic Th2 and mast cells for the control of food allergy. J. Immunol. 179, 1577-1585 (2007).

85. Knight, A.K., Blazquez, A.B., Zhang, S., Mayer, L.F., Sampson, H.A. \& Berin, M.C. CD4T cells activated in the mesenteric lymph node mediate gastrointestinal food allergy in mice. Am. J. Physiol. Gastrointest. Liver Physiol. 293, G1234-G1243 (2007).

86. Hino, A., Kweon, M.N., Fujihashi, K., McGhee, J.R. \& Kiyono, H. Pathological role of large intestinal IL-12p40 for the induction of Th2-type allergic diarrhea. Am. J. Pathol. 164, 1327-1335 (2004).

87. Hino, A., Fukuyama, S., Kataoka, K., Kweon, M.N., Fujihashi, K. \& Kiyono, H. Nasal IL-12p70 DNA prevents and treats intestinal allergic diarrhea. J. Immunol. 174, 7423-7432 (2005).

88. Takayama, N., Igarashi, O., Kweon, M.N. \& Kiyono, H. Regulatory role of Peyer's patches for the inhibition of OVA-induced allergic diarrhea. Clin. Immunol. 123, 199-208 (2007). 